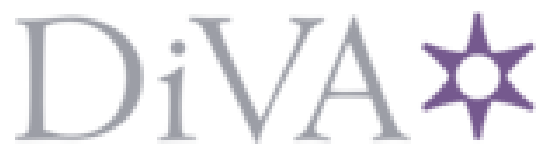

http://www.diva-portal.org

This is the published version of a paper presented at 18th ACM International Conference on Intelligent Virtual Agents, IVA 2018; Western Sydney University's new Parramatta City Campus, Sydney; Australia; 5 November 2018 through 8 November 2018.

Citation for the original published paper:

Ravichandran, N B., Yang, F., Peters, C., Lansner, A., Herman, P. (2018)

Pedestrian simulation as multi-objective reinforcement learning

In: Proceedings of the 18th International Conference on Intelligent Virtual Agents,

IVA 2018 (pp. 307-312).

https://doi.org/10.1145/3267851.3267914

N.B. When citing this work, cite the original published paper.

Permanent link to this version:

http://urn.kb.se/resolve?urn=urn:nbn:se:kth:diva-241487 


\section{Pedestrian simulation as multi-objective reinforcement learning}

\author{
Naresh Balaji Ravichandran* \\ KTH Royal Institute of Technology \\ Stockholm, Sweden \\ nbrav@kth.se
}

\author{
Fangkai Yang ${ }^{\dagger}$ \\ KTH Royal Institute of Technology \\ Stockholm, Sweden \\ fangkai@kth.se
}

\author{
Christopher Peters \\ KTH Royal Institute of Technology \\ Stockholm, Sweden \\ chpeters@kth.se
}

\author{
Anders Lansner \\ KTH Royal Institute of Technology \\ Stockholm, Sweden \\ ala@kth.se
}

\author{
Pawel Herman \\ KTH Royal Institute of Technology \\ Stockholm, Sweden \\ paherman@kth.se
}

\begin{abstract}
Modelling and simulation of pedestrian crowds require agents to reach pre-determined goals and avoid collisions with static obstacles and dynamic pedestrians, while maintaining natural gait behaviour. We model pedestrians as autonomous, learning, and reactive agents employing Reinforcement Learning (RL). Typical RL-based agent simulations suffer poor generalization due to handcrafted reward function to ensure realistic behaviour. In this work, we model pedestrians in a modular framework integrating navigation and collision-avoidance tasks as separate modules. Each such module consists of independent state-spaces and rewards, but with shared action-spaces. Empirical results suggest that such modular framework learning models can show satisfactory performance without tuning parameters, and we compare it with the state-of-art crowd simulation methods.
\end{abstract}

\section{CCS CONCEPTS}

- Computing methodologies $\rightarrow$ Reinforcement learning; Modeling and simulation;

\section{KEYWORDS}

reinforcement learning, agent-based simulation, multi-objective learning, parallel learning

\section{ACM Reference Format:}

Naresh Balaji Ravichandran, Fangkai Yang, Christopher Peters, Anders Lansner, and Pawel Herman. 2018. Pedestrian simulation as multi-objective reinforcement learning. In IVA '18: International Conference on Intelligent Virtual Agents (IVA '18), November 5-8, 2018, Sydney, NSW, Australia. ACM, New York, NY, USA, 6 pages. https://doi.org/10.1145/3267851.3267914

\footnotetext{
${ }^{*}$ This is the corresponding author

${ }^{\dagger}$ This is the corresponding author
}

Permission to make digital or hard copies of all or part of this work for personal or classroom use is granted without fee provided that copies are not made or distributed for profit or commercial advantage and that copies bear this notice and the full citation on the first page. Copyrights for components of this work owned by others than the author(s) must be honored. Abstracting with credit is permitted. To copy otherwise, or republish, to post on servers or to redistribute to lists, requires prior specific permission and/or a fee. Request permissions from permissions@acm.org.

IVA '18, November 5-8, 2018, Sydney, NSW, Australia

(C) 2018 Copyright held by the owner/author(s). Publication rights licensed to ACM. ACM ISBN 978-1-4503-6013-5/18/11 ..\$15.00

https://doi.org/10.1145/3267851.3267914

\section{INTRODUCTION}

Pedestrian crowd simulation has been widely studied in the past decades. The simulation models can be broadly classified into three categories based on spatial resolution [9]: macroscopic, mesoscopic, and microscopic. The macroscopic models $[7,8]$ simulate crowds as fluids in physics, often in large crowd cases, ignoring individual behaviour and interactions. The mesoscopic models are based on cellular automata $[1,2]$, which enable individuals to move in cells, but within fixed and limited directions. The microscopic models $[6,15,17]$, especially the agent-based models, focus on behaviours and movements of each individuals, with the intention of simulating crowd behaviour in a social setting.

In this work, we are interested in agent-based learning models for crowd simulation, where each individual is an intelligent entity, referred as an agent, with decision-making and planning capacity. Specifically, we restrict ourself to agents that are: (1) reactive, i.e., the agents only utilize current sensory information (and any learned values) to make decisions, but cannot plan over an extended period of time; (2) reward-based, where we specify the positive/negative outcomes that the agent should learn to repeat/avoid; (3) decentralized, where the agents cannot examine the decision-strategies of other agents; (4) learn from experience, where the action-selection is completely learned by trial-and-error experience. Learning-based approaches to pedestrian simulation have a particular difficulty in that, when multiple agents are learning behaviour independently and simultaneously, the agents modifying their behaviour pose a non-stationary environment to other agents, leading to a hard learning problem [12]. We argue in this paper that this problem can be mitigated when we approach pedestrian simulation as a multiple objective problem: each agent maximizes navigation towards the goal, while concurrently minimizing collisions.

We employ Reinforcement Learning (RL) framework as the learning and action-selection mechanism for pedestrian simulation. In the RL framework, agents learn by interacting with the environment and change their actions to maximize a reward. RL has a rich history in biological and artificial decision-making, and is widely employed in the field of robotics and machine-learning [13]. RL has also recently found its way to pedestrian simulation. Torrey [14] employed multi-agent RL for crowd simulation in a grid-world environment. Martinez-Gil et al. [12] advocated the use of vector quantization in multi-agent RL, and Godoy et al. [5] combined Optimal Reciprocal Collision Avoidance (ORCA) with RL to efficiently 
explore and learn velocities that would not be feasible in regular ORCA. We introduce a novel RL approach suitable for pedestrian simulation, where we split the learning algorithm into modules that learn separate objectives, and combine them in action-selection. We show that this modular approach is effective in generating plausible pedestrian behaviour.

The rest of this paper will be dedicated to introducing and evaluating this modular RL approach. The paper is organized as follows: Section 2 provides an overview of the current agent-based approaches to pedestrain simulation. Section 3 presents the RL framework, introducing the popular dynamic-programming based solutions to RL, direct policy-search and actor-critic methods. In Section 4, we introduce the model primarily advocated in this paper as a multi-objective modular RL algorithm in the context of pedestrian simulation. Section 5 discusses implementation and experimental details of the model and test scenarios. We present the experimental results of the model in Section 6, and in Section 7 we provide discussions and present future directions for the work.

\section{PEDESTRIAN SIMULATION}

Numerous methods have been proposed to simulate pedestrian crowds that focus on detailed behaviours of individuals in multiagent system. In this section, a general overview of related methods that are used as comparisons is presented. Helbing and Molnar [6] proposed Social Force model which considers each individual as a particle. In this model, each pedestrian has its own force (attraction/repulsive force) which governs behaviours and movements. $\mathrm{Yu}$ et al. [17] proposed Centrifugal model which is also based on particle system. In this work, Social Force model is selected as a representative force model in comparison. Van Den Berg et al. [15] presented Optimal Reciprocal Collision Avoidance (ORCA), which builds on the concept of Reciprocal Velocity Obstacles [16]. It provides collision-free movements by letting each agent shares the responsibility of avoiding pairwise collisions. Also, Karamouzas et al. [10] proposed a predictive collision avoidance model (PCM), which anticipates future collisions in order to avoid them. In Social Force model and Centrifugal model, individuals avoid collisions when they are close, which causes unrealistic movements and even leads to deadlock. In contrast, ORCA anticipate upcoming collisions and lead to collision-free motions.

\section{REINFORCEMENT LEARNING}

The $\mathrm{RL}$ problem is denoted by a finite Markov Decision Process (MDP) and is defined by the tuple $\langle\mathcal{S}, \mathcal{A}, \mathcal{T}, \mathcal{R}, \gamma\rangle$ where: $\mathcal{S}$ is a finite set of states, $\mathcal{A}$ is a finite set of actions, $\mathcal{T}: \mathcal{S} \times \mathcal{A} \rightarrow \mathcal{S}$ is the deterministic transition function, $\mathcal{R}: \mathcal{S} \times \mathcal{A} \times \mathcal{S} \rightarrow \mathbb{R}$ is the reward function and, $\gamma \in[0,1)$ is the discount factor. The state represents the knowledge that the agent possesses about the environment and is expected to be Markovian - encoding all the relevant information of the past, so that the agent only needs to know the current state. In addition to the state, the agent also receives a reward signal. The reward is a scalar number and it quantifies the 'goodness' for the agent. The action, as the name suggests, is performed by the agent on the environment that leads to changes in its state. In addition to the terms introduced in the problem definition, we introduce the following terms that will be useful in the discussion about solving reinforcement learning: $\pi: \mathcal{S} \times \mathcal{A} \rightarrow[0,1]$ is the policy learnt and followed by the agent, denoting the probabilistic mapping from states to actions, $v: \mathcal{S} \rightarrow \mathbb{R}$ is the value function, denoting the long-term desirability of the state.

The agent maximizes the sum of discounted future rewards $\sum_{k=0}^{\infty} \gamma^{k} r\left(s_{k}, a_{k}, s_{k+1}\right)$, called the return, where $\gamma$ is the discount factor, $0 \leq \gamma<1$, which quantifies the importance of temporallydistant rewards. With $\gamma=0$, only the immediate state can be rewarding, while with $\gamma=1$, rewards at all states in the future are equally rewarding. The estimates of returns can be computed for each state, called the value or $v^{\pi}(s)$ for each $s \in \mathcal{S}$.

$$
v^{\pi}(s)=\mathbb{E}\left[\sum_{k=0}^{\infty} \gamma^{k} r\left(s_{k}, a_{k}, s_{k+1}\right) \mid s_{0}=s, a \sim \pi(\cdot \mid s)\right]
$$

where $\mathbb{E}[\cdot]$ is the expected value, and $\pi(\cdot)$ denotes the policy probability distribution.

We can find a general solution to RL by: (1) parametrizing the value-function and policy, and (2) finding the parameters that maximize the value function for each state. This allows framing RL as an optimization problem and approximate it by stochastic gradientdescent (SGD) methods. The value function parametrized by $w$ can be written as $v(s ; \boldsymbol{w})$, and the policy parametrized by $\boldsymbol{\theta}$ can be written as $\pi(a \mid s ; \boldsymbol{\theta})$. The SGD update equation can be written as:

$$
\begin{aligned}
\boldsymbol{w}_{t+1} & =\boldsymbol{w}_{t}+\alpha \delta_{t} \boldsymbol{\nabla}_{\boldsymbol{w}} v\left(s_{t} ; \boldsymbol{w}_{t}\right) \\
\boldsymbol{\theta}_{t+1} & =\boldsymbol{\theta}_{t}+\alpha \delta_{t} \boldsymbol{\nabla}_{\boldsymbol{\theta}} \log \pi\left(a_{t} \mid s_{t} ; \boldsymbol{\theta}_{t}\right)
\end{aligned}
$$

where $\alpha$ is the learning rate and $\delta_{t}=r\left(s_{t}, a_{t}, s_{t+1}\right)+\gamma v\left(s_{t+1} ; \boldsymbol{w}_{t}\right)-$ $v\left(s_{t} ; \boldsymbol{w}_{\boldsymbol{t}}\right)$ is the reward-prediction error. The policy-distribution that selects actions is called the actor, and the value-function that provides information about the state is called the critic, and Eqs. 1 and 2 provide the update equation to learn the actor and the critic respectively. The actor-critic network directly parametrizes both the value-function and policy, which proves to be favourable for the modular architecture that we discuss below.

\section{METHODS}

In this section, we introduce the main model for pedestrian crowd simulation proposed in this paper. Consider a set $\mathcal{P}$ of circular pedestrian agents with diameter $d$ in a given 2D environment with static (or constant velocity) obstacles $O$, with each agent having a fixed set of goal positions. Each agent $p \in \mathcal{P}$ can be represented by a state function $s_{p}(t)=\left\{\vec{x}_{p}(t), \vec{v}_{p}(t)\right\}$, consisting of the agent's position $\vec{x}_{p}$ and velocity $\vec{v}_{p}$ vectors at time $t$. The learning objective is to enable each agent to take actions with this given sensory information, independent of other agents' action strategy, while reaching goals as quickly as possible and avoiding collisions with other agents and obstacles (the set $O \cup \mathcal{P} \backslash p$ ). All agent have, except for their goal position, identical notations and learning algorithms (discussed later). Hence, for convenience, we drop the subscript $p$ for the agent, $s(t)=\{\vec{x}(t), \vec{v}(t)\}$, and discuss the learning for one agent treating other agents and obstacles as included in the obstacle set $\tilde{O} \doteq O \cup \mathcal{P} \backslash p$.

We begin the model formulation by reminding that the pedestrian simulation is treated as a multi-objective problem, with the agent simultaneously and independently (1) maximizing the reaching of goals, and (2) avoiding collisions. We therefore assign each 
objective a module to perform learning and select actions continuously. The modules have a pre-defined state-space ideal for that objective, and a shared action-space. The reward function for both the modules are independently designed. Therefore, the model definition consists of defining (1) the state and (2) reward functions of each module, (3) learning rule, and (4) action-selection mechanism (Fig. 2 for illustration).

State-space: For the module handling goal-directed navigation task, denoted here on as goal, the state information $s_{\text {goal }}$ required for the navigation of agent towards the goal is simply the position of the agent $s_{\text {goal }} \doteq \vec{x}(t)$. This state feature-space required for $\mathrm{RL}$ can be constructed by a set of Gaussian radial basis functions, called place-cells (Fig. 1.a), covering the state-space with centres at $\left\{\vec{x}_{p c}^{i} \mid i \in \mathbb{Z}_{+}, i<N_{p c}\right\}$. The corresponding feature vector can be written as $\phi_{\text {goal }}=\left(\phi_{\text {goal }}^{1}, \ldots, \phi_{\text {goal }}^{N_{p c}}\right)^{T}$, with:

$$
\phi_{\text {goal }}^{i} \doteq \exp \frac{-\left\|\vec{x}-\vec{x}_{p c}^{i}\right\|^{2}}{2 \sigma_{p c}^{2}}
$$

where $\|\cdot\|$ is the $L^{2}$-distance, and $\sigma_{p c}$ is the variance of the Gaussian function. The place-cells are placed throughout the reachable sections of the arena. The value of feature $\phi_{\text {goal }}^{i}$ encodes the probability of finding the agent in the place-cell $i$ location, and the feature values decay exponentially as we move away from $\vec{x}_{p c}^{i}$, with $\sigma_{p c}$ controlling the spread of the feature-values.

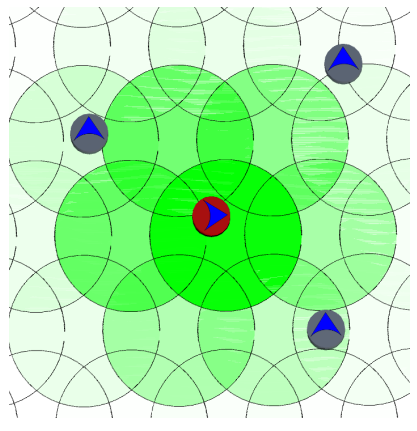

(a)

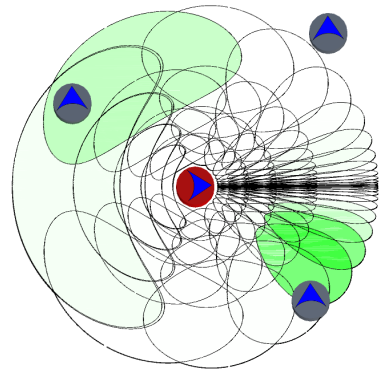

(b)
Figure 1: Pedestrian simulation environment with cylindrical agents (red is of interest) indicating the state featurevalues, (a). place-cells seen as circles on the arena, valued from 0 (white) to 1 (green) based on the agent's position, (b). collision-cells seen as contour lines around the agent, valued from 0 (white) to 1 (green) by the feature-value of obstacles' position relative to the agent.

For the module handling collision-avoidance, denoted hereon as collide, the state-space requires information on the relative position of obstacles that are in the vicinity of the agent and potentially lead to collision. The features of collide module are chosen to be: (1) ego-centric to the agent, (2) finer resolution in the frontal field of view compared to rear, (3) finer resolution for obstacles closer to the agent compared to those farther. The feature are designed, similar to the goal module, using collision-cells $\left\{\vec{x}_{c c}^{i j} \mid i, j \in\right.$ $\left.\mathbb{Z}_{+}, i<N_{c c}^{r}, j<N_{c c}^{\theta}\right\}$. The collision-cells are placed around the agent exponentially less likely as we move away from the agent, and away from the agent's forward direction. The collision-cell position is given by the polar coordinates $\vec{x}_{c c}^{i j}=\left(r_{c c}^{i j}, \theta_{c c}^{i j}\right)^{T}$ where $r_{c c}^{i j} \doteq \frac{\beta_{c c}^{i}}{\beta_{c c}^{N_{c c}^{r}}} v_{\max }$ and $\theta_{c c}^{i j} \doteq \frac{\beta_{c c}^{\left|j-0.5 N_{c c}^{\theta}\right|}-1}{\beta_{c c}^{0.5 N_{c c}^{\theta}}-1} \operatorname{sgn}\left(j-0.5 N_{c c}^{\theta}\right) \pi$, where $|\cdot|$ and $\operatorname{sgn}(\cdot)$ denote the absolute value and sign functions, and the hyper-parameter $\beta_{c c} \in \mathbb{R}_{+}$controls the stretch of the collision-cells around the agent both radially and tangentially. An illustration of the above collision-cells placement around the agent can be seen in Fig. 1.b. We use Gaussian elliptical distance function placed along radial and tangential axes to ensure that the distance measures around the agent are smoothly covered. The feature-vector then can be written as $\phi_{\text {collide }}=\left(\phi_{\text {collide }}^{1}, \ldots, \phi_{\text {collide }}^{N_{c c}^{r} N_{c c}^{\theta}}\right)^{T}$, with:

$$
\begin{aligned}
\phi_{\text {collide }}^{i j}(s) & =\max _{o \in \tilde{O}} \mathcal{N}\left(\left[\begin{array}{l}
r_{o} \\
\theta_{o}
\end{array}\right] \mid\left[\begin{array}{c}
r_{c c}^{i j} \\
\theta_{c c}^{i j}
\end{array}\right],\left[\begin{array}{cc}
\operatorname{var}^{i j}[r] & 0 \\
0 & \operatorname{var}^{i j}[\theta]
\end{array}\right]\right) \\
& =\max _{o \in \tilde{O}} \exp \left\{-0.5 \frac{\left(r_{o}-r_{c c}^{i j}\right)^{2}}{\operatorname{var}^{i j}[r]}-0.5 \frac{\left(\theta_{o}-\theta_{c c}^{i j}\right)^{2}}{\operatorname{var}^{i j}[\theta]}\right\}
\end{aligned}
$$

where $o \in \tilde{O}$ is the obstacle located by the polar coordinates $\left(r_{o}, \theta_{o}\right)$, and $\operatorname{var}^{i j}[r]$ and $\operatorname{var}^{i j}[\theta]$ are the radial and tangential variances, chosen to be average of their neighbouring cell variances, i.e., $\operatorname{var}^{i, j}[r]=0.5\left(\operatorname{var}^{i-1, j}[r]+\operatorname{var}^{i+1, j}[r]\right)$ and $\operatorname{var}{ }^{i, j}[\theta]=$ $0.5\left(\operatorname{var}^{i, j-1}[\theta]+\operatorname{var}^{i, j+1}[\theta]\right)$, to ensure smooth coverage of featurevalues around the agent.

Reward function. The reward function of the modules are straightforward, owing to having tasks allocated independently to each module. The reward function of goal module for an action is defined as the distance traversed towards the goal by taking that action:

$$
r_{\text {goal }}\left(s_{t}, a_{t}, s_{t+1}\right)=\gamma\left\|\vec{x}(t+1)-\vec{x}_{\text {goal }}\right\|-\left\|\vec{x}(t)-\vec{x}_{\text {goal }}\right\|
$$

where $\vec{x}_{\text {goal }}$ is the position vector of the goal pertaining to the agent. The reward function of the collide module is -1 when any of the obstacles is within distance $d$ to the agent:

$$
r_{\text {collide }}\left(s_{t}, a_{t}, s_{t+1}\right)= \begin{cases}-1, & \text { if } \exists o \in \tilde{O}:\left\|\vec{x}(t+1)-\vec{x}_{o}\right\|<d \\ 0, & \text { otherwise }\end{cases}
$$

Action-space: The action-space is identical for all the modules, and denotes the agent's velocity $\vec{v}(t)$ in the allocentric reference frame. For convenience of the learning algorithm, we discretize this space into $N_{a}^{\theta}$ uniformly distributed radial directions and $N_{a}^{r}$ uniformly divided speed values (capped at $v_{\max }$ ). The agent executes the action by first rotating towards the action direction, and translating forward for the action speed. The action feature-vector $\Omega \in \mathbb{R}^{N_{a}^{\theta} N_{a}^{r}}$ is an one-hot encoding represented by 1 for discrete action taken, and 0 otherwise.

Learning. We have formulated the state with the intent of having a probabilistic interpretation of the features. The state-action feature-vector $\psi$ can be defined as $\psi=\operatorname{vec}\left(\phi^{T} \cdot \Omega\right)$, where $\operatorname{vec}(\cdot)$ is the vectorization of the matrix. With this, we can employ a linear function-approximation for the value-function $v(s ; \boldsymbol{w})=\boldsymbol{\phi}(s)^{T} \cdot \boldsymbol{w}$, and a softmax function (linear-exponential) for the policy over the actions $\pi(a \mid s ; \boldsymbol{\theta})=\operatorname{softmax} \boldsymbol{\psi}(s, a)^{T} \cdot \boldsymbol{\theta}$. The learning update rules 
(Eqs. 1 and 2) of actor-critic architecture can be written for our parametrization as:

$$
\begin{aligned}
\boldsymbol{w}_{t+1} & =\boldsymbol{w}_{t}+\alpha \delta_{t} \boldsymbol{\phi}\left(s_{t}\right) \\
\boldsymbol{\theta}_{t+1} & =\boldsymbol{\theta}_{t}+\alpha \delta_{t}\left(\boldsymbol{\psi}\left(s_{t}, a_{t}\right)-\left\langle\boldsymbol{\psi}\left(s_{t}, \cdot\right)\right\rangle\right)
\end{aligned}
$$

where $\langle\psi(s, \cdot)\rangle=\sum_{b} \pi(b \mid s) \psi(s, a)$ is defined as the average feature value weighted by the policy. The update step is applied individually to the modules at every time step based on the reward received for each module.

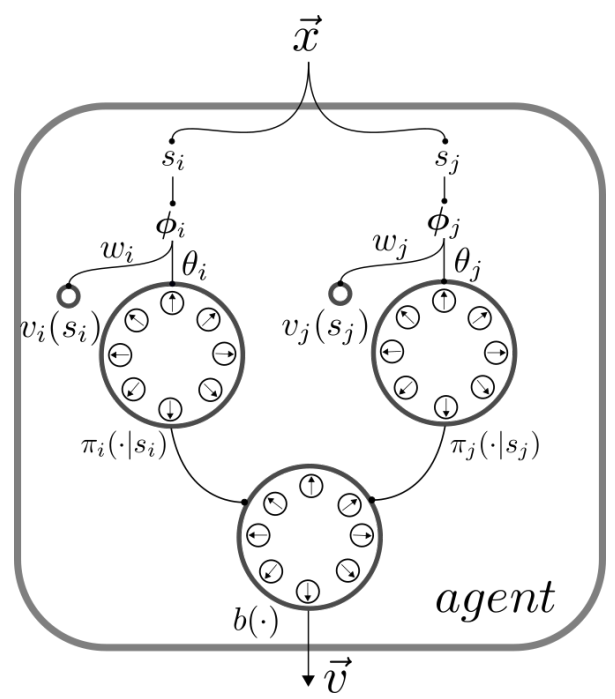

Figure 2: Model architecture for generic modules $i$ and $j$. Each module is represented by the state features $\phi$, critic network $v(s)$ and, actor network $\pi(\cdot \mid s)$. The behavioural policy combines the policy suggested by the actors of all modules, and outputs the action executed by the agent.

Action-selection. Each module $i$ promotes a policy distribution $\pi(\cdot \mid s)$ over actions, which are then combined to a behavioural policy $b(\cdot)$, and subsequently into an action (as a velocity vector). We had deliberately chosen the action-space to be identical for all modules, so that policies can be readily combined. After rotation of collide policy (due to egocentricity of the module), we combine the modules multiplicatively: $b(a) \propto \prod_{i} \pi_{i}(a \mid s)$, and choose softmax actions from our behavioural policy.

\section{IMPLEMENTATION}

\subsection{Architecture}

We implemented the reinforcement-learning based pedestrian simulation by splitting the model into two parts: (1) multi-agent physics simulation, and (2) modular RL system. All simulations were run on Intel Core i5-5200U CPU at $2.20 \mathrm{GHz}$. Unity 3 D game engine ${ }^{1}$ was used to create testing scenarios, simulate pedestrian physics and visualize simulation on personal computer (PC). The reinforcement learning model of this paper was implemented in $\mathrm{C}++$, and was parallelized with Message Passing Interface (MPI) for each agent (modules within agents were run on a single process).

\footnotetext{
${ }^{1}$ https://unity3d.com/
}

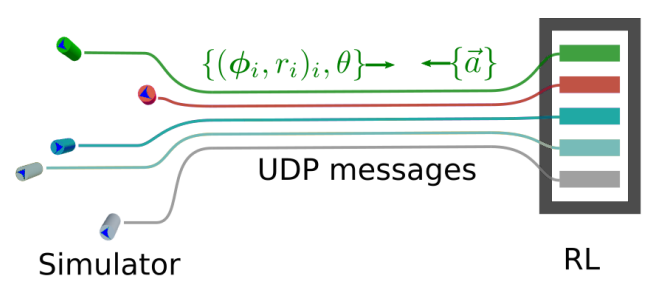

Figure 3: Setup and interface of simulation environment and action-selection/learning system

Communication between Unity3D project and the RL system is setup through UDP channels, of which, one is dedicated to each agent. The UDP messages are communicated synchronously each update time-step $\Delta$ of RL. In each time-step, Unity $3 D$ sends the feature vector and reward value of the agent for both goal and collide modules. Noting that the feature vector is sparse-coded valued between 0 and 1 , we set the threshold of feature-values to 0.05 in order to efficiently transmit messages. The messages are received by the RL system, updated, and action value are sent back for each agent. During learning phase (Sec.6.1), the simulation is run at $25 \mathrm{x}$, and at all other times run at $1 \mathrm{x}$. The code used to generate the results presented here is made available as a repository on Github at https://github.com/nbrav/clever-walkers.

\subsection{Scenarios}

We set up five different scenarios as shown in Fig. 4 with different number of agents, some of them with obstacles. The scenarios include: (a). bottleneck: 15 agents are placed near a narrow exit and will escape through this exit (Fig. 4.a); (b). circle: 16 agents are placed on a circle and will walk to their antipodal goals (Fig. 4.b); (c). intersection: two groups of 8 agents walk perpendicularly and meet in the middle of the crossing (Fig. 4.c); (d). hallway: two groups of 4 agents walk in opposite directions and reach their antipodal goals in open area (Fig. 4.d); (e). bi-directional: two groups of 8 agents walk to reach their antipodal goals in a narrow corridor (Fig. 4.e). The agents in the scenarios are modelled as cylinders with radius $0.5 \mathrm{~m}$, preferred speed $1.34 \mathrm{~m} / \mathrm{s}$, and maximal speed $2 \mathrm{~m} / \mathrm{s}$.

\section{EVALUATION}

We present the evaluation of the proposed method in two parts: (1) evaluating the learning and stability of the modular RL algorithm, and (2) comparing the performance of the method against popular (non-learning) models specialized for pedestrian simulation. The list of parameters used for pedestrian simulation (in Unity $3 D$ ) is provided in Table 1, and the parameters used in the RL algorithm are provided in Table 2 .

We compare our model with Social Force (SF) model and ORCA model, and set maximal neighbours to consider as 10, mass as 80 and, maximal distance to take other agents into account in navigation as $5 \mathrm{~m}$. Specific to ORCA, the time horizon with respect to other agents is set as $3 \mathrm{~s}$, and the time horizon with respect to obstacles is set as 

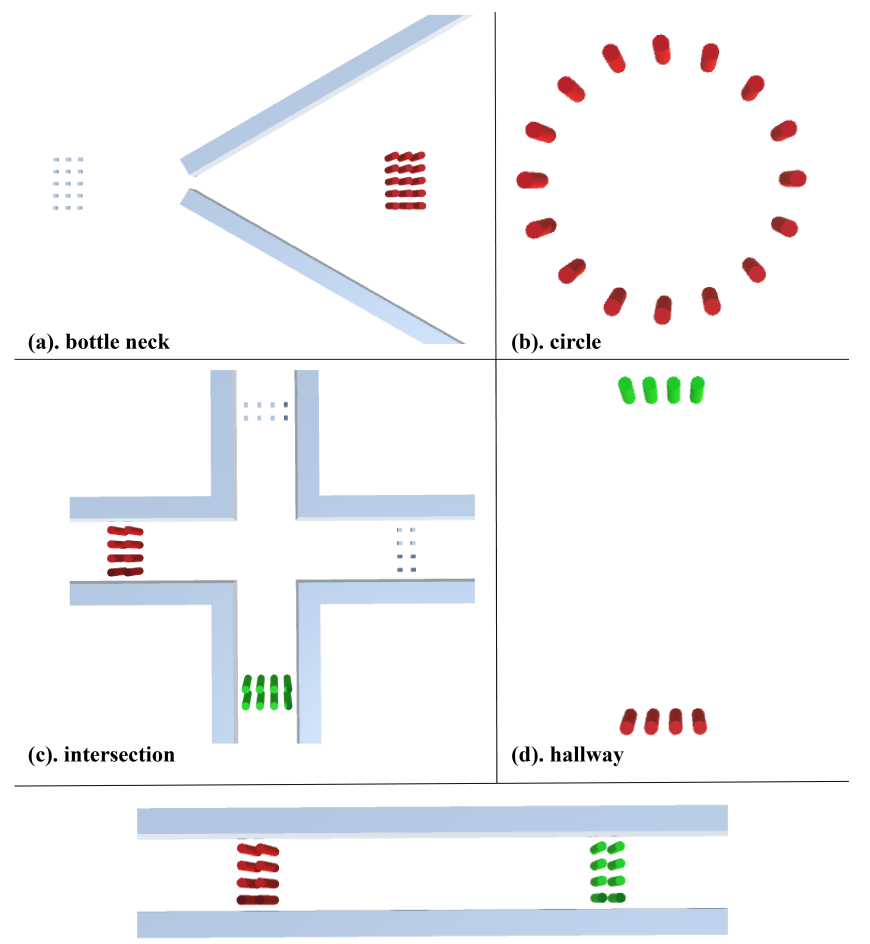

(e). bi-directional

Figure 4: Evaluation scenes

Table 1: Simulation environment parameters

\begin{tabular}{lll}
\hline Name & Description & Value \\
\hline$\Delta$ & update time-step $(\mathrm{s})$ & 0.5 \\
$T$ & trial duration $(\mathrm{s})$ & 100 \\
$d$ & agent diameter $(\mathrm{m})$ & 0.5 \\
$v_{\max }$ & maximum velocity $(\mathrm{m} / \mathrm{s})$ & 2 \\
$N_{p c}$ & no. place-cells & 225 \\
$\sigma_{\mathrm{goal}}$ & place-cell variance & 2.0 \\
$N_{c c}^{r}$ & no. collision-cells radially & 30 \\
$N_{c c}^{\theta}$ & no. collision-cells tangentially & 4 \\
$\beta_{c c}$ & base-rate & 1.5 \\
$N_{a}^{r}$ & no. discretized action speed & 3 \\
$N_{a}^{\theta}$ & no. discretized action direction & 8 \\
\hline
\end{tabular}

$0.15 s$ (refer to RVO2 library ${ }^{2}$ for more details on the parameters). SF, ORCA, and PCM are implemented in the Menge ${ }^{3}$ [3] framework and implemented in Unity. The Unity implementation ${ }^{4}$ provides more detailed information on the parameters.

\subsection{Learning and convergence}

Convergence of parameters is of paramount importance for learning methods. Vanilla actor-critic methods provide theoretical guarantees for convergence to optimal value-function and policies [11].

\footnotetext{
${ }^{2} \mathrm{http}: / /$ gamma.cs.unc.edu/RVO2/documentation/2.0/

${ }^{3} \mathrm{http}: / /$ gamma.cs.unc.edu/Menge/

${ }^{4}$ https://github.com/keanudicap/CrowdSimulateStateOfArt
}

Table 2: Reinforcement Learning parameters

\begin{tabular}{lll}
\hline Name & Description & Value \\
\hline$\alpha$ & learning rate & 0.01 \\
$\gamma$ & discount factor & 0.95 \\
\hline
\end{tabular}

However, for the modular approach introduced in this work with multiple objectives, no such guarantee exist. Here, we test the model empirically for convergence for both goal and collide modules. We chose the cumulative reward through the course of a trial, averaged over all agents, as an indicator of convergence (as RL aims to maximize cumulative reward).

For brevity, we demonstrate the convergence results to the circle scene (Fig. 5). The cumulative reward for the goal module (left) monotonically increases, and converges at the maximal value. Conversely, the collide module has a surge of collisions at the beginning, just when goal receives high rewards by learning trajectories cutting through the centre of the circle, leading to collisions from similar-acting agents. The collide module then, learns to avoid cutting through agents, and discovers alternate paths around other pedestrians. Fig. 6 shows sampled trajectories of 5 randomly selected agents before (left) and after (right) the learning phase. At the beginning of the simulation, the agents randomly explore random actions. At the end of the learning phase, agents converges to distinct behavioural policies, some of which learn direct paths towards the goal (trajectories orange and green), while some learn indirect paths (brown and blue).

The above evaluation indicates that the learning phase was successful. However, convergence of cumulative reward does not necessarily imply the agent is behaving optimally. In fact, optimality within multi-objective setting is not easily defined as singleobjective optimization, as we cannot directly compare independent objectives. In the next evaluation method, we rather, compare directly the performance of our model with the pedestrian simulation method.
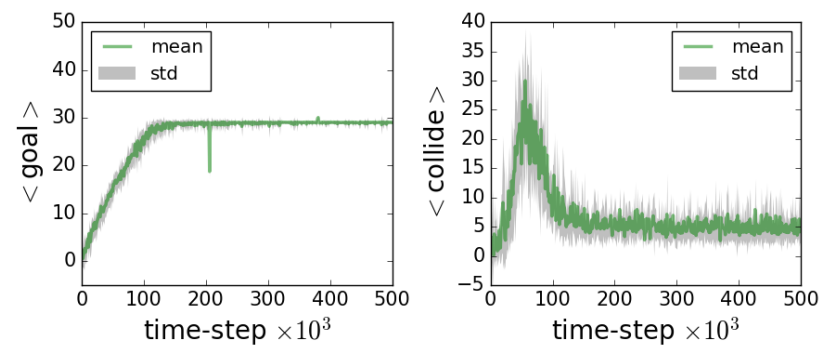

Figure 5: Learning curve for circle scene

\subsection{Comparison with pedestrian simulation methods}

We compare the proposed method with SF, ORCA, and PCM under two evaluation metrics: (a) time to goal, which, as the name suggests, is the simulation time (in seconds) taken for the agent to navigate from the starting position to the goal position (both positions are shown for all scenarios in Fig. 4), and (b) number of collisions, that each agent experiences in the trial while reaching for the goal. Both 


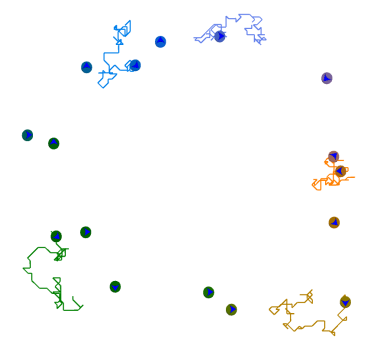

(a) before learning

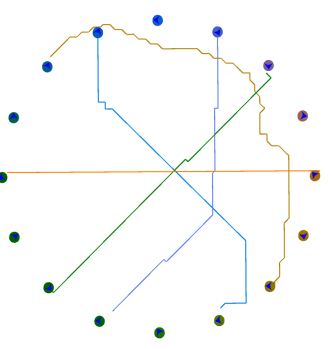

(b) after learning
Figure 6: Trajectory followed by 5 randomly selected agents in scene circle (a). before and (b). after learning

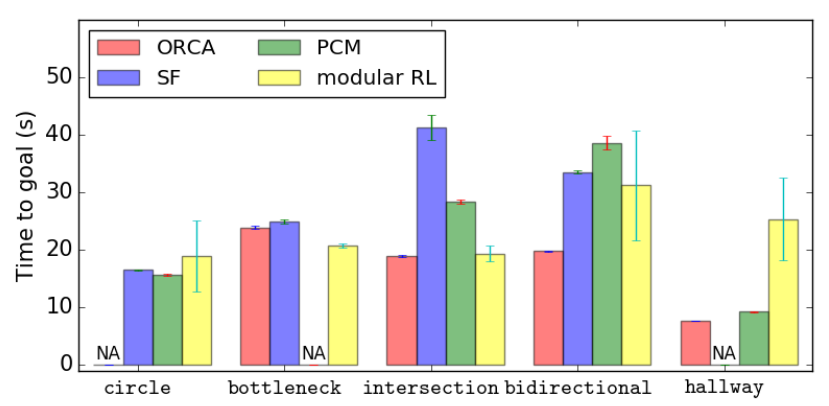

Figure 7: Time to reach goal (s)

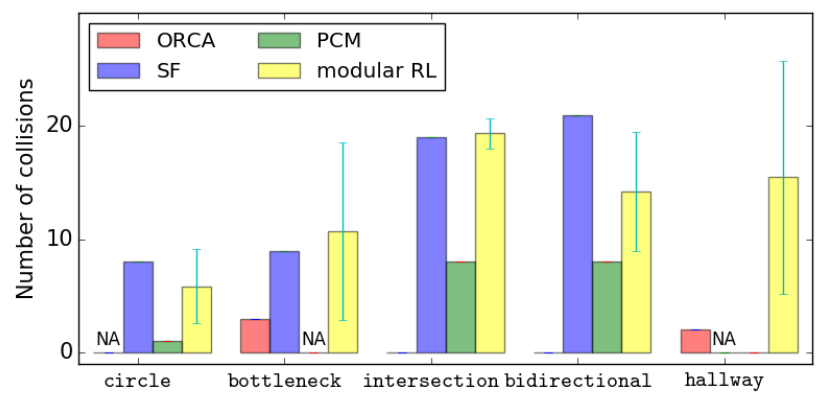

Figure 8: Number of collisions

the metrics are averaged over the agents present in the scenario, and averaged over 25 trials. We start the evaluation of our model after $500 \times 10^{3}$ update time-steps, i.e., upon convergence (Section 6.1). Results are presented in Fig. 7 and 8.

Overall, the proposed modular RL method performs comparably with other pedestrian simulation methods, and surpasses all other methods in the bottleneck scene when evaluated by the time to goal. ORCA failed in circle scene where agents got stuck in the middle (similar failed ORCA case was shown in [4]). In terms of number of collisions, our model is comparable with Social Forces method in most scenes. However, ORCA and PCM perform consistently better than our model, which is unsurprising, given both are anticipatory collision avoidance systems. Additionally, the modular RL method has very high standard deviation compared to all other methods in almost all scenes. This was an expected behaviour from our model for two reasons: (1) the agents learn very different policies, that may lead to same agents constantly performing worse and vice verse, (2) the policies themselves can be stochastic, which could cause variability within trials of the same agent. We can see this as a consequence of the flexibility offered by learning agents in changing their behaviour.

\section{DISCUSSION AND CONCLUSIONS}

In this paper, we introduced a learning model for pedestrian simulation. Three key aspects were taken into consideration when designing the model: (1) generalizability across different configurations of the environment, (2) act as a minimal and natural extension to the RL framework, (3) keeping the parameters interpretable and at a minimum. The key contribution of this work is the identification of pedestrian simulation as a multi-objective problem, and approaching it using modular RL. We opine that modular representation of task policy holds a powerful approach at handling multiple tasks, as opposed to directly tuning the reward function based on requirements. Compared with other simulation methods, our model doesn't need to tune parameters to avoid failure and shows its scalability in different scenes. There are many promising directions to carry the work forward, such as adding modular representation of prior information, representing idiosyncratic agent nature, and evaluating the scalability of the model in high-density crowd, and many more.

\section{REFERENCES}

[1] Victor Blue and Jeffrey Adler. 1998. Emergent fundamental pedestrian flows from cellular automata microsimulation. Transportation Research Record: fournal of the Transportation Research Board 1644 (1998), 29-36.

[2] Victor Blue and Jeffrey Adler. 2000. Cellular automata model of emergent collective bi-directional pedestrian dynamics. In Proc. Artificial Life VII. 437-445.

[3] Sean Curtis, Andrew Best, and Dinesh Manocha. 2016. Menge: A Modular Framework for Simulating Crowd Movement. Collective Dynamics 1, 0 (2016), 1-40. https://doi.org/10.17815/CD.2016.1

[4] Julio Godoy, Tiannan Chen, Stephen J Guy, Ioannis Karamouzas, and Maria Gini. 2017. ALAN: Adaptive Learning for Multi-Agent Navigation. arXiv preprint arXiv:1710.04296 (2017).

[5] Julio E Godoy, Ioannis Karamouzas, Stephen J Guy, and Maria Gini. 2015. Adaptive learning for multi-agent navigation. In Proceedings of the 2015 International Conference on Autonomous Agents and Multiagent Systems. International Foundation for Autonomous Agents and Multiagent Systems, 1577-1585.

[6] Dirk Helbing and Peter Molnar. 1995. Social force model for pedestrian dynamics. Physical review E 51, 5 (1995), 4282.

[7] Roger L Hughes. 2002. A continuum theory for the flow of pedestrians. Transportation Research Part B: Methodological 36, 6 (2002), 507-535.

[8] Roger L Hughes. 2003. The flow of human crowds. Annual review of fluid mechanics 35, 1 (2003), 169-182.

[9] Kiran Ijaz, Shaleeza Sohail, and Sonia Hashish. 2015. A survey of latest approaches for crowd simulation and modeling using hybrid techniques. In 17th UKSIMAMSS International Conference on Modelling and Simulation. 111-116.

[10] Ioannis Karamouzas, Peter Heil, Pascal Van Beek, and Mark H Overmars. 2009. A predictive collision avoidance model for pedestrian simulation. In International Workshop on Motion in Games. Springer, 41-52.

[11] Vijay R Konda and John N Tsitsiklis. 2000. Actor-critic algorithms. In Advances in neural information processing systems. 1008-1014.

[12] Francisco Martinez-Gil, Miguel Lozano, and Fernando Fernández. 2015. Strategies for simulating pedestrian navigation with multiple reinforcement learning agents. Autonomous Agents and Multi-Agent Systems 29, 1 (2015), 98-130.

[13] Richard S Sutton and Andrew G Barto. 1998. Reinforcement learning: An introduction. Vol. 1. MIT press Cambridge.

[14] Lisa Torrey. 2010. Crowd Simulation Via Multi-Agent Reinforcement Learning.. In AIIDE.

[15] Jur Van Den Berg, Stephen J Guy, Ming Lin, and Dinesh Manocha. 2011. Reciprocal n-body collision avoidance. In Robotics research. Springer, 3-19.

[16] Jur Van den Berg, Ming Lin, and Dinesh Manocha. 2008. Reciprocal velocity obstacles for real-time multi-agent navigation. In Robotics and Automation, 2008. ICRA 2008. IEEE International Conference on. IEEE, 1928-1935.

[17] WJ Yu, R Chen, LY Dong, and SQ Dai. 2005. Centrifugal force model for pedestrian dynamics. Physical Review E 72, 2 (2005), 026112. 